\title{
Simulation of micro-scale porous flow using smoothed particle hydrodynamics
}

\author{
$\begin{array}{lll}\text { P. Hassard } & \text { I. Turner } & \text { T. Farrell } \\ & \text { T } & \text { D. } \text { Lester }^{4}\end{array}$
}

(Received 13 March 2015; revised 24 February 2016)

\begin{abstract}
Fluid flow in a porous medium is a well-studied aspect of applied mathematics with significant real-world application. The standard modelling approach for this type of flow is to homogenise the porous structure. A dual-scale model, with the smaller scale at the pore-scale, would possibly capture the fluid mechanical phenomena more faithfully than a volume averaged approach. We investigate the significance of the microstructure shape on the flux through the medium. We also evaluate whether smoothed particle hydrodynamics may be viable in a dual-scale model. We find that varying the shape of the porous structure causes the average flux to vary significantly. This contradicts the assumption commonly made that only the porosity is important. We conclude that there is significant information present in the dualscale model that is lost by a volume averaged model. We also find that
\end{abstract}

http://journal.austms.org.au/ojs/index.php/ANZIAMJ/article/view/9408 gives this article, (c) Austral. Mathematical Soc. 2016. Published March 21, 2016, as part of the Proceedings of the 17th Biennial Computational Techniques and Applications Conference. ISSN 1446-8735. (Print two pages per sheet of paper.) Copies of this article must not be made otherwise available on the internet; instead link directly to this URL for this article. 
the smoothed particle hydrodynamics simulation is computationally intensive, but that there is a time-saving measure that may provide viability to the dual-scale model.

\section{Contents}

1 Introduction

C464

2 Background

C466

3 Implementation

$\mathrm{C} 470$

4 Results

C473

5 Conclusions

C476

References

C476

\section{Introduction}

Multiphase transport phenomena play an integral role in many branches of engineering and applied mathematics. The macroscopic approach for modelling heat and mass transport in heterogeneous porous media typically uses volume averaging as an upscaling method to overcome the difficulties associated with quantifying transport within the complex geometry of the underlying pore network [22]. In this upscaling method, volume-averaged equations, where the averaged volume contains on the order of hundreds or thousands of pores, are used to derive conservation laws that resemble those of a continuum, except for the appearance of volume-averaged quantities and effective parameters. A potential difficulty of this approach is the requirement to specify the nonlinear effective parameters. One such parameter of interest 
is relative permeability, which arises from Darcy's law:

$$
\boldsymbol{v}=-\frac{\mathrm{K}}{\mu}(\nabla \mathrm{P}-\rho \mathbf{g}),
$$

where $\boldsymbol{v}$ is the fluid velocity, $\boldsymbol{\mu}$ is the dynamic viscosity, $\mathrm{P}$ is the pressure, $\rho$ is the density, $\mathbf{g}$ is gravitational acceleration, and $\mathrm{K}$ is the effective permeability, a product of the fluid-independent intrinsic permeability $k$ and the relative permeability $k_{r}$. Darcy's law results when volume averaging techniques are applied to the Navier-Stokes equations [21].

Homogenisation theory is often used to predict effective parameters from the microstructure of the porous medium $[18,9]$. Under the assumption that the underlying pore structure consists of a periodic arrangement of cells, homogenisation theory results in steady-state, uncoupled problems that must be solved on a specified unit cell prior to simulation [2]. However, there are configurations where this type of scaling approach fails. This provides the perfect motivation for moving towards a completely coupled, dual-scale modelling approach. Dual-scale models seek to couple the volume-averaged scale interdependently with the scale of the porous microstructure by imposing suitably defined boundary conditions on the microscopic problem [19, 4]. This strategy avoids the need to define macroscopic quantities, such as relative permeability, that are challenging to define and often difficult to determine experimentally.

The formulation of the dual-scale model follows the model of Alyaev et al. [1]. At the macro-scale, the finite volume method is used to solve the continuity equation for conservation of mass:

$$
\nabla \cdot \mathbf{q}_{M}=\mathrm{S},
$$

where $\mathbf{q}_{M}$ is the macro-scale fluid velocity and $\mathbf{S}$ is a source term. To connect the scales, the gradient of pressure across a macro-scale control volume face is imposed on the pore-scale model located at the face. This pore-scale model is solved for the pore-scale velocity $\mathbf{q}_{\mathfrak{m}}$, which is averaged over the pore space 
to give the macro-scale velocity normal to the face:

$$
\mathbf{q}_{M} \cdot \mathfrak{n}=\frac{1}{V_{T}} \int_{\Omega_{f}} \mathbf{q}_{m} \cdot \mathfrak{n} \mathrm{d} \Omega,
$$

where $\Omega_{\mathrm{f}}$ is the fluid region of the pore-scale domain $\Omega, V_{\mathrm{T}}$ is the total volume of $\Omega$, and $\mathfrak{n}$ is the unit normal to the control volume face. This feedback mechanism between macro-scale and pore-scale can also accommodate other types of transport, such as solute transport, which will be explored in future research.

Several models are of interest for the micro-scale (pore-scale), but here we limit our focus to smoothed particle hydrodynamics (SPH). Section 2 briefly reviews the SPH method. With the dual-scale model as context and motivation, we investigate whether the shape of the porous structure affects the average flux, in order to evaluate the usefulness of such a model. Section 3 discusses how we implemented SPH in this context and Section 4 presents our results. We also discuss the viability of a dual-scale model in terms of how practical (i.e., fast) the SPH runtime is. This is important since in the dual-scale model, we intend to run many of these simulations - one at each macro-scale control volume face, at each time-step.

\section{Background}

The method of SPH was first developed to model stellar fluid dynamics [6, 8], but it has since been applied to the incompressible Navier-Stokes equations [15]

$$
\begin{aligned}
& \nabla \cdot \mathbf{q}_{\mathrm{m}}=0, \\
& \frac{\mathrm{d} \mathbf{q}_{\mathrm{m}}}{\mathrm{dt}}=-\frac{\nabla \mathrm{P}_{\mathrm{m}}}{\rho_{\mathrm{m}}}+v \nabla^{2} \mathbf{q}_{\mathrm{m}}+\mathrm{F},
\end{aligned}
$$

where $\mathbf{q}_{m}$ is the fluid velocity, $P_{m}$ is pressure, $\rho_{m}$ is density, $v$ is kinematic viscosity, $\mathbf{F}$ is a body force, and subscript $m$ denotes a pore-scale quantity. 
Only equation (5) is explicitly solved since equation (4) describes conservation of mass which is automatically satisfied in SPH. Incompressibility is modelled by an equation of state (see below).

SPH models a domain as a number of point particles that obey a force balance. Each physical quantity is calculated at a particle by summing the contributions of the neighbouring particles, weighted according to their distance away from the particle of interest:

$$
\langle A\rangle_{a}=\sum_{b} \frac{m_{b}}{\rho_{b}} A_{b} W_{a b}
$$

where $\langle\cdot\rangle$ denotes the SPH representation, $a$ and $b$ represent particles, subscripts $a$ or $b$ represent a quantity's value at particle $a$ or $b, A$ is an arbitrary quantity, $m$ is mass, and $W_{a b}$ is the weight assigned to the pair $a b$ and is a function of their separation distance. For convenience, we drop all $m$ subscripts since in SPH we only consider pore-scale quantities.

Derivatives of quantities may be calculated by simply operating the derivative on the weighting kernel:

$$
\langle\nabla A\rangle_{\mathrm{a}}=\sum_{\mathrm{b}} \frac{\mathrm{m}_{\mathrm{b}}}{\rho_{\mathrm{b}}} \mathrm{A}_{\mathrm{b}} \nabla_{\mathrm{a}} \mathrm{W}_{\mathrm{ab}} .
$$

However, this approach can lead to non-conservative forces. For example, Monaghan [10] found that, when calculating the pressure gradient term of the Navier-Stokes equations (5), the above derivative form did not conserve momentum. Monaghan instead proposed the explicitly conservative form which we use here:

$$
\left\langle\frac{\nabla P}{\rho}\right\rangle_{a}=\sum_{b} m_{b}\left(\frac{P_{a}}{\rho_{a}^{2}}+\frac{P_{b}}{\rho_{b}^{2}}\right) \nabla_{a} W_{a b} .
$$

Additionally, when equation (7) is applied to a second derivative (replacing $\boldsymbol{\nabla}$ with $\nabla^{2}$ ), the result is sensitive to disorder in the arrangement of particles [10]. 
An alternate form widely used is $[3,13]$

$$
\langle\nabla \cdot(\lambda \nabla A)\rangle_{a}=\sum_{b} \frac{m_{b}}{\rho_{b}}\left(\lambda_{a}+\lambda_{b}\right)\left(A_{a}-A_{b}\right) F_{a b},
$$

where we define $\nabla_{a} W_{a b}=r_{a b} F_{a b}$ with $r_{a b}$ the vector from particle $b$ to particle $a$. The scalar quantity $\lambda$ is an arbitrary coefficient which can represent such quantities as diffusivity, conductivity and viscosity. We use equation (9) to discretise the viscous term in equation (5).

Whilst different weighting kernels $W$ have been used, they are generally Gaussian or similar in shape. To avoid summing unnecessarily many minuscule contributions from far away particles, kernels with compact support are preferred [13]. The most common are cubic and quintic B-splines that approximate the Gaussian [16, 20]. Lower order splines have Fourier transforms that decrease slowly and so are vulnerable to instability from transverse modes [14].

We must consider the arrangement of particles, both because we need to create an initial condition and also to be wary of unstable configurations. A regular hexagonal lattice arrangement is a stable configuration for the particles in SPH [16]. This configuration has the highest possible number of equidistant nearest neighbours. If particles are too close together, then SPH encounters pairing instability wherein a pair of close particles merge, thereby decreasing the resolution [16].

Whilst SPH was originally designed for compressible fluids, an artificial equation of state is implemented so that the fluid remains nearly incompressible [10]. This equation of state is often the Tait equation [15, 13, 20]

$$
\mathrm{P}=\frac{\mathrm{c}^{2} \rho_{0}}{\gamma}\left[\left(\frac{\rho}{\rho_{0}}\right)^{\gamma}-1\right]
$$

where $\rho_{0}$ is a reference density, $\mathrm{c}$ is an artificial speed of sound, and $\gamma$ is a numerical parameter controlling the sensitivity of pressure $P$ to density fluctuations $\left|\rho-\rho_{0}\right| / \rho_{0}$. The two most widely used values are $\gamma=7$ and 
$\gamma=1$ [15]. We use $\gamma=1$ and neglect the -1 in (10) so that the Tait equation reduces to the simple linear form [5]

$$
\mathrm{P}=\mathrm{c}^{2} \rho .
$$

The value chosen for the speed of sound $c$ is a compromise between quasiincompressibility and a manageable time-step. The recommended value is such that the Mach number is $\sim 0.1[10]$. This keeps density fluctuations below $1 \%$, and allows a reasonably large time-step.

Another option is to exactly impose incompressibility by solving a Poisson equation [17]. At each time-step the fluid is allowed to evolve without considering pressure, allowing density fluctuations to grow. Since the exact fluctuations are known, a Poisson equation can be solved for the pressure distribution that will reverse these fluctuations. The effects of this pressure distribution are retroactively imposed. This method allows for much smaller density fluctuations, but involves significant additional computation associated with solving an additional linear system at each time-step. Morris et al. [15] argue that the SPH interpolation is only accurate to within approximately $1 \%$, rendering the value of smaller density fluctuations minimal.

To integrate the system of ordinary differential equations that is generated by SPH, an explicit scheme can be used with a sufficiently small time-step [15, 20]. Implicit SPH schemes also exist $[11,7]$ but, for simplicity, we use an explicit scheme in this work.

Since SPH is a Lagrangian framework it uses the material derivative, which avoids the nonlinear advection term present in the Eulerian framework. SPH also automatically satisfies conservation of mass if the particles exchange no mass [13]. However, boundary conditions are difficult to implement, especially those involving a normal vector, since they cannot be imposed at particular points in space but must be imposed on particles which could be anywhere. To impose boundary conditions in SPH, artificial particles are created on the boundary or on the other side of the boundary compared to the fluid particles. These solid particles, or boundary particles, are given attributes similar to the 
fluid particles, but in most cases are not affected by these attributes - only the fluid particles are affected.

A no-slip condition is imposed to first order by simply moving the boundary particles according to the relevant boundary velocity. A higher order condition is given by extrapolating fluid velocity past the boundary to the artificial particles' positions and assigning this artificial velocity to boundary particles [15].

A solid wall boundary must also have a no-penetration condition. One method of implementing this condition is to assign to the boundary particles a force that repels fluid particles and which is inversely proportional to the distance between the boundary and fluid particles [13]. Another method is to attribute pressure to the boundary particles, as if they were fluid particles, so that they repel actual fluid particles without the need to explicitly specify a boundary force [17]. In simple geometries, this second approach generates less fluid disturbance [13].

\section{Implementation}

We are interested in using SPH for our dual-scale model because particles move with the flow field (avoiding the need to specify a mesh), the more convenient material derivative is used (the $\mathbf{d} \mathbf{q} / \mathbf{d} t$ in equation (5) as opposed to the Eulerian form $\partial \mathbf{q} / \partial \mathbf{t}+\mathbf{q} \cdot \boldsymbol{\nabla} \mathbf{q}$ ), and conservation laws are inherent or simple to enforce [13]. As a test problem we choose pressure-driven incompressible flow of a single fluid through a square lattice of infinitely long solid cylinders [15]. The cylinders are arranged periodically and so the problem is simplified to a two-dimensional unit cell with periodic boundary conditions. This problem is relevant to our dual-scale model since it is a crude representation of the micro-structure of a two-dimensional porous medium with the non-solid area representing porosity and the velocity representing the volumetric flux. 
We firstly simulated circular cylinders, but to investigate the effect of cylinder shape on average flux we also simulated arrays of different cylinder shapes. We chose cylinder cross-sections that produce distinctly different channels for fluid flow. To form long, open channels we chose an ellipse cross-section with eccentricity $\sqrt{3} / 2$ oriented parallel to the flow. To form channels that periodically restrict flow into narrow regions we again chose an ellipse crosssection with eccentricity $\sqrt{3} / 2$, but oriented perpendicular to the flow. To form channels with flat surfaces and corners we choose the approximately square hyperellipse cross-section $x^{10}+y^{10}=a^{10}$.

In problems where fluid enters and leaves the domain SPH is forced to reallocate memory in order to create and annihilate particles during the simulation. However, the periodicity in our simulations eliminates this difficulty-for every particle that leaves the domain, there enters another with identical properties.

The unit cell for the cylinder array is square, with a single circular/elliptical/ hyperelliptical inclusion in the centre. We assume the inclusion to be unaffected by the fluid, with no-slip and no-penetration conditions on its surface. We model the pressure gradient via a body force, constant in space and time. This represents a macro-scale pressure gradient which remains constant on the small spatial and temporal scales of the micro-scale domain.

Only the steady-state behaviour of the system is important since we assume the large ratio of the scales to imply that the micro-scale reaches equilibrium quickly compared to the macro-scale time-scale. Because of this, we compare simulations using a static initial condition with those using an initial condition that approximates the steady state, expecting the same steady-state result but different amounts of computational effort.

We used Matlab to implement our solution method, which involved using the SPH method to spatially discretise the Navier-Stokes equations (5) and the (explicit) forward Euler method for temporal discretisation. We imposed several conditions on the time-step to ensure stability [15]. For the reasons outlined in Section 2, to define the pressure derivative and viscosity term 
in (5) we used equations (8) and (9), respectively. Due to the stability concern discussed in Section 2, we used a quintic B-spline for the SPH kernel, and following the suggestion of Morris et al. [15], we set the smoothing length to be 1.5 times the initial particle spacing.

Preliminary simulations, describing the incompressibility with the Tait equation (10), its linear version (11), or a Poisson equation, found that the Poisson method took several times longer to run than the other two, without a significant corresponding improvement in accuracy. The Tait and linear methods produced similar results, so we used the slightly faster linear method.

Initially we placed the particles on a hexagonal lattice as close to regular as possible within the periodic square domain. This was an attempt to maintain a somewhat stable configuration. Morris et al. [15], who studied circular cross-section cylinders, simply defined particles that fell inside the circle as solid and those that fell outside as fluid, but this means that the boundary does not faithfully resolve the true boundary (i.e. the circle). To resolve the true boundary as well as possible, we placed solid particles on the boundary. To avoid stability problems to do with particles being too close together, we also deleted any particle within a quarter particle spacing of the boundary. This disrupted the original mesh, where each particle has the same volume around it. To ensure that density was initially constant across the domain we calculated the volume around each particle (the reciprocal of the sum of the kernel values $\left.\sum_{b} W_{a b}\right)$ and assigned to it a corresponding mass.

At the cylinder boundary, to apply the no-slip condition we used the higher order condition which requires extrapolation across the cylinder boundary, as described in Section 2, and to treat the no-penetration condition we artificially maintained pressure in the solid particles. Both of these methods were chosen for their higher accuracy compared to other discussed methods. To invoke periodic boundary conditions we calculated the particle positions modulo the domain length, and also considered duplicate particles nearby in the neighbouring unit cells [12]. 
Figure 1: Velocity magnitude contours (left, with units $1.74 \times 10^{-5} \mathrm{~m} / \mathrm{s}$ ) and pressure contours (right, with units $10^{-6} \mathrm{~Pa}$ ) for the cylindrical case.
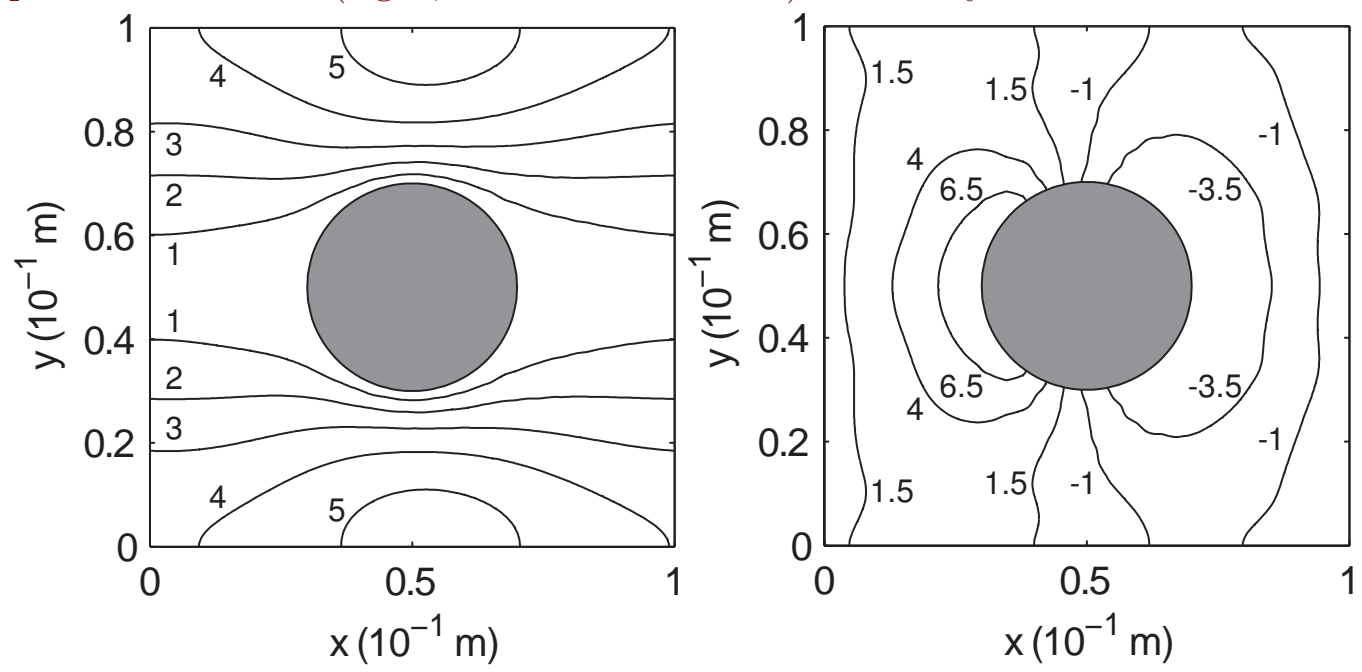

\section{Results}

To compare the results of our simulation with Morris et al. [15], we use the parameters from their model of flow with Reynolds number equal to one. As well as performing an SPH simulation, Morris et al. solved the problem using the finite element method applied to incompressible Stokes flow. In their model the unit cell is aligned with the $x y$ plane and the force simulating a pressure gradient acts in the positive $x$ direction. The velocity and pressure contours from our SPH cylinder simulation (see Figure 1) recover the results of Morris et al. up to a multiplicative or additive constant. We find that the given time-step constraints did not ensure stability, possibly due to the artificially increased effect of viscosity at the boundary.

Having verified that the velocity and pressure fields from our cylinder simulation exhibit the behaviour we expect, we produced velocity fields for the elliptic and approximately square cylinders (see Figure 2). We find that the 
Figure 2: Velocity magnitude contours for the ellipse parallel to the flow (left), ellipse perpendicular to the flow (right) and square (bottom), with units $1.74 \times$ $10^{-5} \mathrm{~m} / \mathrm{s}$.
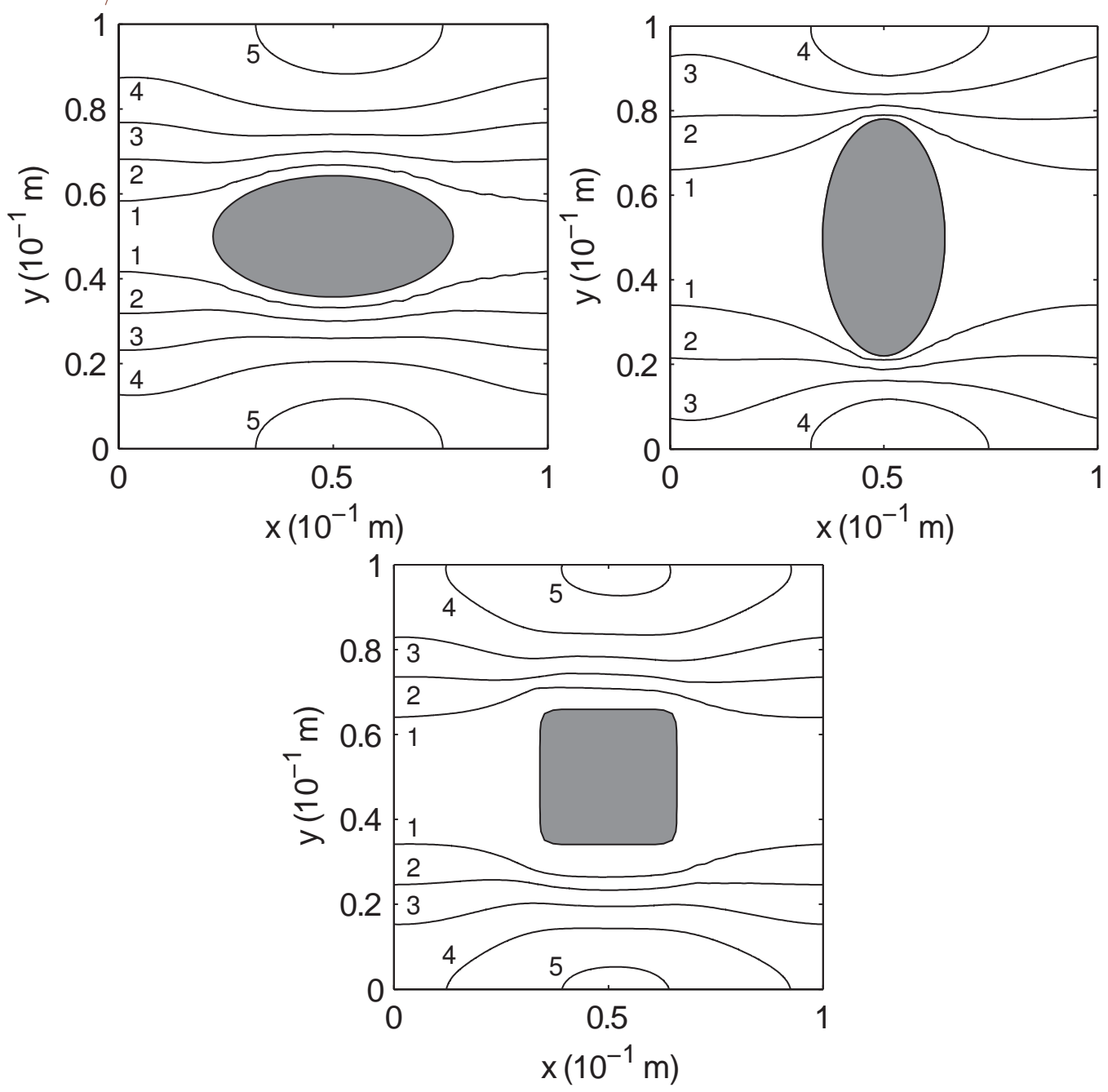
fluid moves more quickly the farther it is from the cylinder. This means that faster flow is possible in the wider channels between ellipses positioned parallel to the flow, and that the flow is restricted by the narrower channels between ellipses positioned perpendicular to the flow. Additionally, we find that fluid heading directly towards or away from the cylinder is slow. This means that the flat edges of the approximately square cylinders restrict velocity more than the circular cylinders.

We compare the fluid velocity across the cylinder shapes in order to quantify the effect of shape. Compared to the mean horizontal velocity for the circle, the mean horizontal velocity was $\sim 15 \%$ larger for the parallel ellipse, $\sim 30 \%$ smaller for the perpendicular ellipse, and $\sim 10 \%$ smaller for the square. We kept the cross-sectional area constant across all four shapes so that porosity was identical in each case. Additionally, for the two elliptic cases we kept not only the porosity constant but also the shape, and this led to roughly a $60 \%$ larger mean horizontal velocity for the parallel case compared to the perpendicular case. This is important for a dual-scale model since it tells us that the flux depends not only on the porosity of the micro-structure but also significantly on its shape and its orientation relative to the flow.

For all of the cylinder shapes, when we used a rough estimate of the steady state flow (merely a parabola in y) as the initial condition, the solution converged to the same steady state as for a static initial condition, and it did so several times faster. In a dual-scale model, this convergence could be exploited by using the steady-state solution from each micro-scale problem as the initial condition at the subsequent time-step. In our example, we used a very rough estimate of the steady state and still managed to achieve upwards of five times speed-up. In contrast, the steady-state solution will undergo relatively small changes between time-steps in a dual-scale model. We expect that this strategy will provide much more speed-up in a dual-scale context. Currently, with nearly 3000 particles, our code takes the order of 10 minutes to reach steady state in Matlab R2014a on an Intel Core i7-4800MQ $2.70 \mathrm{GHz}$ processor. The code would take significantly less time to run in a dual-scale model, but would not necessarily be viable. In an attempt to make it viable, 
we could implement further efficiency measures or simulate fewer particles.

\section{Conclusions}

We investigated the potential for SPH as the micro-scale model in a dualscale model of a porous medium. Our research led to two main findings. Firstly, the flux through a domain depends significantly on the shape and orientation of the porous structure, not simply the porosity. This suggests that the dual-scale approach will recover information lost when performing volume averaging, and so would be worthwhile. Secondly, while our current code is computationally expensive, there are time-saving approaches that will potentially make this method viable.

In future work we intend to embed SPH into a three-dimensional dual-scale modelling framework and compare it against experimental results. In this context we also intend to compare the suitability of SPH with other methods, such as the lattice Boltzmann or boundary element methods.

Acknowledgements We acknowledge ANZIAM and CSIRO for their funding and support through the CTAC student support scheme. We also acknowledge CSIRO for their funding and collaboration as part of the IDTC program. Finally, we thank the reviewers for valuable feedback that guided improvements to the manuscript.

\section{References}

[1] S. Alyaev, E. Keilegavlen, and J. M. Nordbotten. Analysis of control volume heterogeneous multiscale methods for single phase flow in porous media. Multiscale Model. Sim., 12(1):335-363, 2014. doi:10.1137/120885541 C465 
[2] H. Brenner. Dispersion resulting from flow through spatially periodic porous media. Phil. Trans. R. Soc. A, 297(1430):81-133, 1980. doi:10.1098/rsta.1980.0205 C465

[3] L. Brookshaw. A method of calculating radiative heat diffusion in particle simulations. Proc. Astron. Soc. Aust., 6(2):207-210, 1985. http://adsabs. harvard.edu/abs/1985PASAu ...6 . 207B. C468

[4] E. J. Carr and I. W. Turner. Two-scale computational modelling of water flow in unsaturated soils containing irregular-shaped inclusions. Int. J. Numer. Meth. Eng., 98(3):157-173, 2014. doi:10.1002/nme.4625 $\mathrm{C} 465$

[5] A. J. Chorin. A numerical method for solving incompressible viscous flow problems. J. Comput. Phys., 2(1):12-26, 1967. doi:10.1016/0021-9991(67)90037-X C469

[6] R. A. Gingold and J. J. Monaghan. Smoothed particle hydrodynamics: Theory and application to non-spherical stars. Mon. Not. R. Astron. Soc., 181:375-389, 1977. doi:10.1093/mnras/181.3.375 C466

[7] S. Litvinov, M. Ellero, X. Y. Hu, and N. A. Adams. A splitting scheme for highly dissipative smoothed particle dynamics. J. Comput. Phys., 229(15):5457-5464, 2010. doi:10.1016/j.jcp.2010.03.040 C469

[8] L. B. Lucy. A numerical approach to the testing of the fission hypothesis. Astron. J., 82(12):1013-1024, 1977. doi:10.1086/112164 C466

[9] C. C. Mei, J. L. Auriault, and C.-O. Ng. Some applications of the homogenization theory. Adv. Appl. Mech., 32:278-348, 1996. doi:10.1016/S0065-2156(08)70078-4 C465

[10] J. J. Monaghan. Smoothed particle hydrodynamics. Annu. Rev. Astron. Astrophys., 30:543-574, 1992. doi:10.1146/annurev.aa.30.090192.002551 C467, C468, C469 
[11] J. J. Monaghan. Implicit SPH drag and dusty gas dynamics. J. Comput. Phys., 138(2):801-820, 1997. doi:10.1006/jcph.1997.5846 C469

[12] J. J. Monaghan. From stars to volcanoes: The SPH story. In Eduardo Ramos, Gerardo Cisneros, Rafael Fernández-Flores, and Alfredo Santillán-González, editors, Computational Fluid Dynamics, Proceedings of the Fourth UNAM Supercomputing Conference, pages 193-203, Singapore, 2001. World Scientific. doi:10.1142/4623 C472

[13] J. J. Monaghan. Smoothed particle hydrodynamics. Rep. Prog. Phys., 68:1703-1759, 2005. doi:10.1088/0034-4885/68/8/R01 C468, C469, C470

[14] J. P. Morris. A study of the stability properties of smooth particle hydrodynamics. Publ. Astron. Soc. Aust., 13:97-102, 1996. http://adsabs.harvard.edu/abs/1996PASA...13...97M. C468

[15] J. P. Morris, P. J. Fox, and Y. Zhu. Modeling low Reynolds number incompressible flows using SPH. J. Comput. Phys., 136(1):214-226, 1997. doi:10.1006/jcph.1997.5776 C466, C468, C469, C470, C471, C472, C473

[16] D. J. Price. Smoothed particle hydrodynamics and magnetohydrodynamics. J. Comput. Phys., 231(3):759-794, 2012. doi:10.1016/j.jcp.2010.12.011 C468

[17] S. Shao and E. Y. M. Lo. Incompressible SPH method for simulating Newtonian and non-Newtonian flows with a free surface. Adv. Water Resour., 26(7):787-800, 2003. doi:10.1016/S0309-1708(03)00030-7 C469, $\mathrm{C} 470$

[18] R. E. Showalter. Microstructure models of porous media. In Homogenization and Porous Media, pages 183-202. Interdisciplinary Applied Mathematics. Springer New York, 1997. doi:10.1007/978-1-4612-1920-0_9 C465

[19] A. Szymkiewicz, J. Lewandowska, R. Angulo-Jaramillo, and J. Butlariska. Two-scale modeling of unsaturated water flow in a 
double-porosity medium under axisymmetric conditions. Can. Geotech. J., 45:238-251, 2008. doi:10.1139/T07-096 C465

[20] P. van Liedekerke, B. Smeets, T. Odenthal, E. Tijskens, and H. Ramon. Solving microscopic flow problems using Stokes equations in SPH.

Comput. Phys. Commun., 184:1686-1696, 2013.

doi:10.1016/j.cpc.2013.02.013 C468, C469

[21] S. Whitaker. Flow in porous media I: A theoretical derivation of Darcy's law. Transport Porous Med., 1(1):3-25, 1986. doi:10.1007/BF01036523 $\mathrm{C} 465$

[22] S. Whitaker. Coupled transport in multiphase systems: A theory of drying. Adv. Heat Trans., 31:1-104, 1998. doi:10.1016/S0065-2717(08)70240-5 C464

\section{Author addresses}

1. P. Hassard, School of Mathematical Sciences, Queensland University of Technology, Brisbane, Queensland 4000, Australia. mailto:patrick.hassard@hdr.qut.edu.au

2. I. Turner, School of Mathematical Sciences, Queensland University of Technology, Brisbane, Queensland 4000, Australia; ARC Centre of Excellence for Mathematical and Statistical Frontiers. mailto:i.turner@qut.edu.au

3. T. Farrell, School of Mathematical Sciences, Queensland University of Technology, Brisbane, Queensland 4000, Australia; ARC Centre of Excellence for Mathematical and Statistical Frontiers. mailto:t.farrell@qut.edu.au

4. D. Lester, School of Civil, Environmental and Chemical Engineering, Royal Melbourne Institute of Technology, Melbourne, Victoria 3000, Australia. 
mailto:daniel.lester@rmit.edu.au 